\title{
REQUIREMENT AREA REVIEW: FLEXIBLE STUDY AREA FOR TRAFFIC IMPACT ASSESSMENTS
}

\author{
PATHIRAJA A.L.A.C. ${ }^{1}$, P.C.P. DE SILVA² \& AMILA B. JAYASINGHE 3 \\ 1,2,3Department of Town \& Country Planning, University of Moratuwa, Moratuwa, Sri Lanka \\ 119806oE@uom.lk, amapathirajauom2018@gmail.com, 2chameerap@uom.lk, \\ chameera.chameera.desilva@gmail.com,3amilabj@uom.lk
}

\begin{abstract}
New developments will contribute to increased traffic to the external environment. Therefore, the majority of nations, in planning and building regulations and guidelines have adopted steps to control them beforehand. Similarly, in Sri Lanka, new developments in the approval process should be undertaken, based on their legal specifications and through a Traffic Impact Assessment (TIA). Although the management of traffic from new developments is exceedingly positive, one of the problematic sectors noted in the TIA study is a fixed study area. Consequently, a $500 \mathrm{~m}$ buffer area from the proposed site edges is currently being taken as the study area in Sri Lanka. The geographical extent of the traffic impact area is not a fixed bound-ary. Particularly based on a set of parameters, the true catchment area could be changed. A fixed boundary may, therefore, misdirect developers, consultants and planning authorities in decision making in situations of transport planning. Owing to this very debatable selection, almost all studies have been incorrect. Consequently, this research focuses on investigating the requirement of flexible study areas for traffic impact assessments of distinctly unique developments.
\end{abstract}

Keywords: Flexible Study Area, TIA, Traffic Impact, Traffic Impact Area, Traffic Impact Assessment

\section{Introduction}

Urban road traffic congestion is a dangerous global issue existing in every major city (Wen, Chin, \& Lai, 2017). A new development, thus, essentially attracts vehicle demand for the accessible and adjacent roads and linked junctions in the vicinity (Weerasekera, 2011). Therefore, new constructions or alterations to the existing development or renovations has an impact on the extent of external traffic (Land Transport Authority, 2011). If not properly diagnosed, challenges would become increasingly severe (Ponnurangama\&Umadevib, 2014). Therefore, once this was identified, numerous countries have taken different planning and building regulations and guidelines to detect and eliminate adverse effects beforehand (Cooley, Gruyter, \&Delbosc, 2016). A Traffic Impact Assessment (TIA) is needed to carry out according to the legal conditions, to obtain a development permit for new developments. It identifies potential traffic impacts from the proposed development for the surrounding road network and provides corrective action to minimise them (Teodoro\&Regidor, 2005).

Mega constructions in Sri Lanka too, have to submit a traffic impact assessment as laid down by the parking \& traffic control (Regulation 34, Schedule III) under the City of Colombo Development Plan for development permits from 1986 onwards. While there is an enormous positive movement, questionable areas may still emerge in some areas referred to the Term of Reference (TOR) in traffic impact assessments (Director, Enforcement Division of Urban Development Authority, 2019). Delineating the true catchment area for traffic impact analysis is one of the debatable areas. The 50om buffer zone from site edges is currently taken as the study area for traffic impact assessments. This is a fixed figure similar to most other countries (Ponnurangama\&Umadevib, 2014). However, the area of influenced traffic after a new development varies with different indicators (Abley, Durdin, \& Douglass, 2010).

Even though there are numerous different TIA guidelines in most countries, a flexible boundary for transport impact analysis of the TIA is not well understood through the guidelines, or even at practice (Cooley, Gruyter, \&Delbosc, 2016). Besides, the guidelines are included in a reasonable study area, depending on the type and scope of the development, especially in the United States. In the TIA guidelines and the TORs, a minimum study area limit should be recommended as the initial (Weller, 2007). Therefore, the flexible boundary limit should be clearly defined (Cooley, Gruyter, \&Delbosc, 2016) and to understand how much the new development will affect the environment, a geographical demarcation of the traffic impact area is needed (Wen, Chin, \& Lai, 2017). If not, due to this very arbitrary selection, many findings would be erroneous. In addition, 
numerous experts in the transport field believe that it is essential for increasing development to have an acceptable catchment area for the evaluation of traffic impacts. Therefore, this research objects to investigate the requirement of a flexible study area for traffic impact assessments of each unique development.

\section{Literature Review}

Unanticipated growth is a massive concern since traffic in most countries is unbalanced (Azra\&Hoque, 2014). Therefore, some kind of serious transport studies, which are commonly known as the Traffic Impact Assessment (TIA), should be carried out before the approval for construction (May, et al., 2019). Different countries and different researchers use a variety of names for this, such as transport assessment, transport impact assessment, transportation assessment, traffic impact assessment, traffic impact study, traffic report and integrated transport assessment (ITA) (Abley, Durdin, \& Douglass, 2010). With certain mitigation actions and directives, this study may be rejected or accepted.

The vehicle demand from each new construction activity is usually increased in the neighbouring road network (Khade, Khode, \&Bhakhtyapuri, 2017). Thus, the accumulative vehicle trip attraction from new developments will generate many adverse impacts on the street network (Ponnurangama\&Umadevib, 2014). The extent of traffic impact should, therefore, be correctly estimated from any new construction of the adjacent roads and intersections. Unless properly managed, serious planning and transportation problems can arise (Abley, Durdin, \& Douglass, 2010). Therefore, most cities have undertaken a traffic impact study to approve new developments and improvements to existing developments (May, et al., 2019). The TIAs is used to seek an impact on all aspects of the transportation system due to new developments (Sarkar, Maitri, \& Joshi, 2015). Therefore, this assessment is more cost-effective and time-efficient to make future development decisions at the planning stage (Cooley, Gruyter, \&Delbosc, 2016). The required inputs and its scope should be clearly described under the TIA study to make a sustainable transport system (Abley, Durdin, \& Douglass, 2010). Accordingly, the study area or impact area is the base to conduct any impact study (Cooley, Gruyter, \&Delbosc, 2016). Therefore, in a TIA study, the most important and most difficult stage is the delineation of the study area, which is also called the "impact area" (Abley, Durdin, \& Douglass, 2010).

Nevertheless, planning and building approving agencies of most countries such as Bangladesh (Azra\&Hoque, 2014), Philippines (Teodoro\&Regidor, 2005), Canada (Engineering and Capital Infrastructure Services Infrastructure, Development \& Enterprise, April 2016), China (Weller, 2007) and United Arab Emirates (Department of Transport, November 2009) etc., have failed to identify a proper mechanism to delineate the study area for the transport study. For generalization, most agencies both local and international have instructed TIA consultants to undertake a certain study area, irrespective of the development type and scale. As an example, the Land Transport Division of the Ministry of Public Infrastructure \& Land Transport in Malaysia, through the TIA Guideline in November, 2015 instructs that the study area should be a 1km radius adjacent to the site. Likewise, impact levels to all the access roads, nearby roads and junctions within $1 \mathrm{~km}$ radius from edge of the site should be studied as the direct traffic impact area under the guidelines in Mauritius (Ministry of Public Infrastructure \& Land Transport, November 2015). In this instance, there is a fixed impact area under these regulations and the consultant may discuss the extent of the study area with the Ministry of Public Infrastructure \& Land Transport, Mauritius. Locally, the Urban Development Authority in Sri Lanka instructs to get a 500m radius buffer area from the site edges as the traffic impact study area (TOR for TIA, 2019).

Many countries such as Malaysia (Ministry of Public Infrastructure \& Land Transport, 2015), Singapore (Land Transport Authority, 2017), Australia (Department of Planning, August 2016), Mauritius (Ministry of Public Infrastructure \& Land Transport, November 2015), New Zealand (Abley, Durdin, \& Douglass, 2010), and United Kingdom (Department for Regional Development (DRD) \& Department of the Environment (DOE), November 2006) etc. also apply a fixed study area for all developments under their own TIA guidelines. Within all the above guidelines, consultants may extend, but have to comply with, the set guideline for the minimum. Some agencies have made a positive movement by requesting the consultants to demarcate study areas 
as per the development type and scale. The boundary of the study areas was clearly outlined in the TIA guidelines in many cities and municipalities in the United States, based on the use and scale of the proposed development (Azra\&Hoque, 2014) such as the State of Utah (Utah Department of Transportation, February 2015), Indiana (see figure 1) (Indiana Department of Transportation, May 2015 ), Arizona (Town Council Of Buckeye, December 2012), Minnesota (New Prague City Council, September, 2010) and City of Visalia (Community Development Department, 2019) etc.

A minimum study area is an essential aspect under the TIA guidelines (Weller, 2007) and it should define the detailed study area limits for all development types (Cooley, Gruyter, \&Delbosc, 2016). Abley, Durdin, \& Douglass (2010), clearly explained that the design years are generally changing by the location, scope, and development type and therefore, it is recommended that there should be at least a 10-year space as "future assessment year". Nevertheless, all demarcations are static and no rigid dynamic ways for demarcation could be observed elsewhere. Abley, Durdin, \& Douglass (2010), have explained that the scope of the TIA study should be described based on the different development types. At the same time, the minimum study area for all developments should be specified according to its use and development size (Cooley, Gruyter, \&Delbosc, 2016). Subsequently, he illustrated that the flexible impact areas should be defined, based on the "scale of activity and the extent of impact". Also, many research studies discuss that the impactable boundary of each development will differ and is not fixed. Therefore, due to variations in travel patterns, socio-economic and cultural conditions, and locational characteristics, the same guideline cannot be applied to all developments (Azra\&Hoque, 2014). Thus, a large number of developments will attract massive traffic around (Yayat K.D., Kombaitan B., Pradono, Purboyo H.P.H., 2015). The bare land available around is also one of the factors to change the impact proportion of the area (Chen Y. \& Liu A., 2019).

According to most scholars, the extent of the true catchment area after a new development, varies with different parameters such as the proposed development type / use (Abley, Durdin, \& Douglass, 2010), size / scale of the proposed development, location of the proposed development (Azra\&Hoque, 2014), scale of activity of the proposed development, around land use pattern (chen y. \&liu a., 2019), modes of transport facilities around, nature of transport network around, trip generation from the proposed development (Cooley, Gruyter, \&Delbosc, 2016), intersection performance around, road link performance around (Weerasekera, 2011),topographical barriers, connectivity of road network, nearby trip production / attraction points, population density around (Sarkar, Maitri, \& Joshi, 2015), prevailing traffic conditions on the existing road systems (Ministry of Public Infrastructure \& Land Transport, 2015), considerable distance from the site, close proximity of new developments to the site (Land Transport Authority, 2017), site access points (PMK Associates, Inc., July 2006), transport related infrastructure developments (Department of Planning, August 2016), pedestrian routes and cycle routes, availability of bare land (chen y. \&liu a., 2019), access road classifications, access road width and speed limits, number of lanes of the access road, walking distances to public transport connections (Engineering and Capital Infrastructure Services Infrastructure, Development \& Enterprise, April 2016), drive distance (Dramowicz, 2005), drive time (Segal, 1998), trading hours (Dolega, Pavlis, \& Singleton, 2015), perception of safety, parking facilities (Wegener \&Fuerst, 2004), travel cost, employment, density, urban density, neighbourhood design ( Leszczyc, Sinha, \&Sahgal, 2004) and accessibility (Delloye, 2018) etc. Hence, a fixed border might also point in the wrong direction for the planning authorities to take transport planning decisions.

\section{Methodology}

This research reviewed 50 TIA reports in Sri Lanka through the cornel method, and they were analyzed to recognize the requirement of a flexible study area or fixed study area for traffic impact assessments of each development. All the TIA reports have been collected from the Urban Development Authority and randomly selected for this study. All reports, prepared by different consultants for a variety of development categories, have been submitted within the past 5 years, to obtain traffic planning clearance for a development permit.

This study used Google Earth Pro Software, Microsoft Excel and IBM SPSS Statistics software. Google Earth Pro Software was used to identify the traffic count locations and to find the direct 
distances from each site edges to traffic count locations. Microsoft Excel and IBM SPSS Statistics software were applied to identify the radius of the adopted impact areas and the gap between the true catchment and adopted catchment in TIA studies.

\section{Analysis and Results}

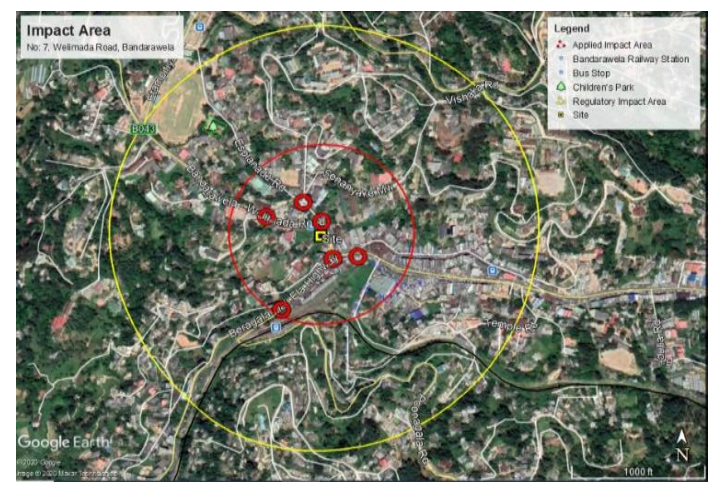

Figure 1: Impact Area - No: 7, Welimada Road,

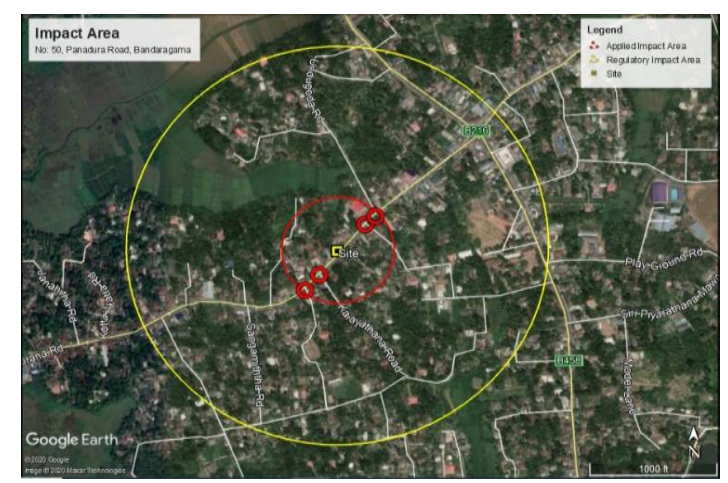

Figure 2: Impact Area - No: 5o, Panadura Road,

All 50 locations of TIA reports are within different local authorities in Sri Lanka. They were taken through a random sampling technique to analyze the need for a flexible study area for TIA studies. These TIA reports are prepared for different development purposes such as retail, shopping, religious, recreational, industrial, residential, office, educational and other utilities etc., and different development scales. Further, the area, where the traffic count locations (traffic data collected and analyzed roads and junctions) in these TIA reports, are considered as the adopted impact area or "Applied Impact Area" at the practice in this study. Moreover, the direct distances in each direction from the site edges to traffic count locations will be measured and the maximum value of the radius will be taken as the radius of the applied impact areas. The 500m radius is the study area or initial impact area under the Urban Development Authority guidelines and regulations for TIAs in Sri Lanka (Urban Development Authority, 2018). Although it is a fixed study area, there is a gap between the regulatory boundary and the adopted boundaries at the practice in TIAs. It is obviously proven as below.

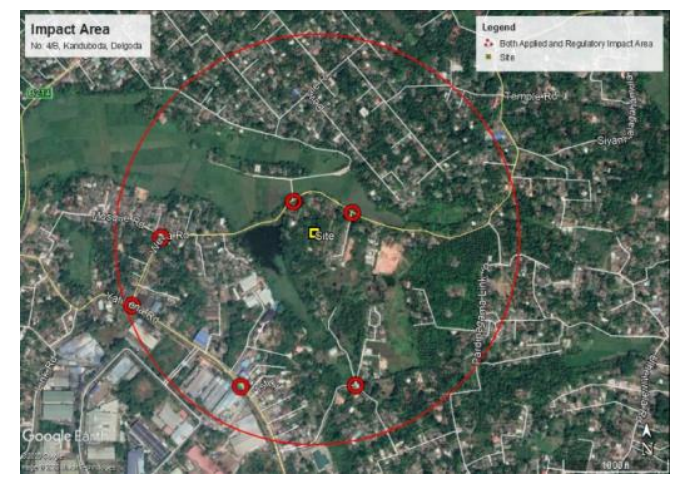

Figure 3: Impact Area - No: 4/B, Kanduboda, Delgoda

The total floor area of the proposed development in Figure 1 is 5,785.16 sqm. The TIA report was prepared on 17th September 2018 and it is now at the operational stage. The boundary of traffic count locations is the practically adopted impact area throughout this TIA study. All the traffic planning decisions have been taken based on this study area. But, the regulated study area (50om radius) is larger than the adopted impact area at the practice. Accordingly, this TIA study has taken a maximum $240 \mathrm{~m}$ radius impact area, which is lesser than the legal requirement. It is clearly shown in Figure 1.

The total floor area of the proposed development in Figure 2 is 1,160 sqm. The TIA report was prepared in December 2017 and it is now at the operational stage. Here also, TIA study has taken a maximum of $130 \mathrm{~m}$ radius impact area and is much less than the legal requirement. It is clearly shown in Figure 2. 


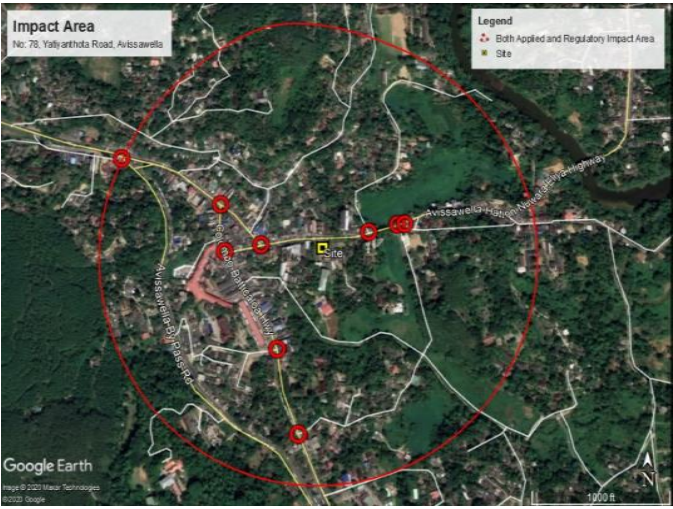

Figure 4: Impact Area - No: 78, Yatiyanthota Road,

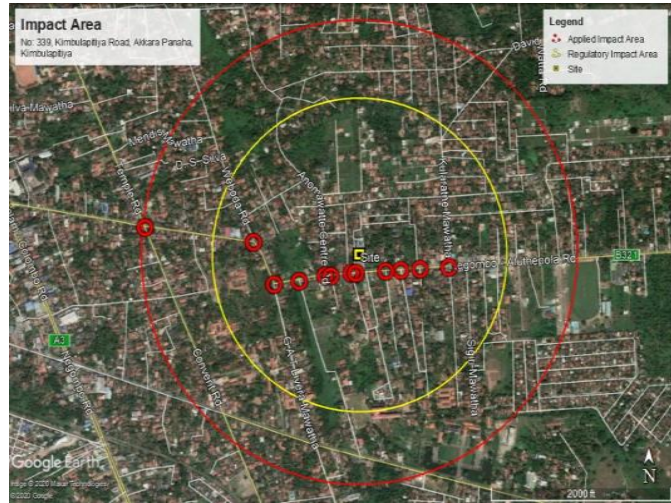
Figure 5: Impact Area - No: 339, Kimbulapitiya Road,

The total gross area of the building in Figure 3 is 15,060 sqm. This TIA study has taken the maximum 500m radius impact area. Therefore, the legally required impact area and the practically adopted impact area are the same boundaries of this TIA study. Accordingly, this study has been carried out following the legal guidelines by the TIA consultant (Figure 3).

The total gross area of the building in Figure 5 is $11,516.89 \mathrm{sqm}$. This TIA study also has taken the maximum 500m radius impact area. Therefore, this study has been carried out following the legal guidelines by the TIA consultant (Figure 5).

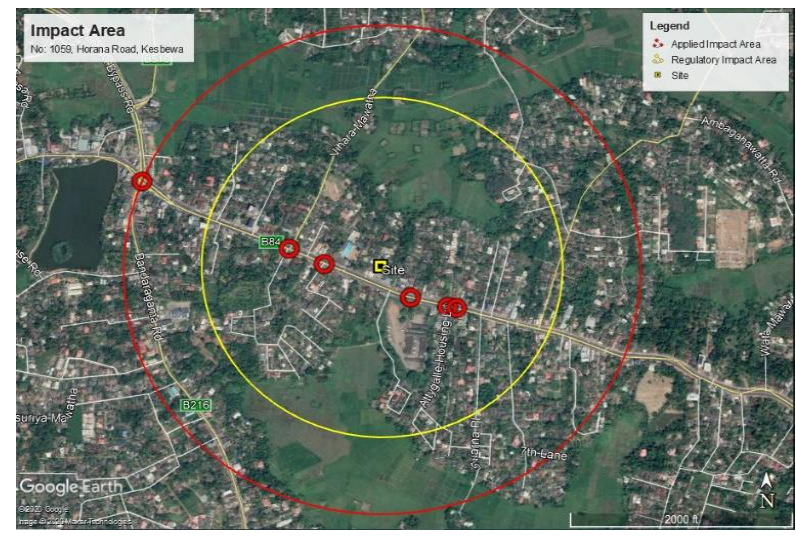

Figure 6: Impact Area - No: 1059, Horana Road, Kesbewa

The total floor area of the proposed development in Figure 4 is $1,151 \mathrm{sqm}$. The TIA report was prepared in February 2018 and is now at the operational stage. This study has taken a maximum of $740 \mathrm{~m}$ radius impact area, and is larger than the legal requirement. It is clearly shown in figure 4 .

The total floor area of the proposed development in Figure 6 is $1,186.0$ sqm. The TIA report was prepared in June 2018 and it is now at the operational stage. This study has taken a maximum of $710 \mathrm{~m}$ radius impact area and exceeds the legal requirement. It is clearly shown in Figure 6, and the table below shows the results of the study in a summary.

Table 1: Results - Radius of the Applied Study Area

\begin{tabular}{|c|c|c|c|c|c|c|}
\hline No. & $\begin{array}{c}\text { GPS } \\
\text { Location }\end{array}$ & Address & $\begin{array}{l}\text { Development } \\
\text { Type }\end{array}$ & $\begin{array}{l}\text { Scale of the } \\
\text { Proposed } \\
\text { Development } \\
\text { (Floor Area - } \\
\text { sqm) }\end{array}$ & $\begin{array}{c}\text { Radius of } \\
\text { the Applied } \\
\text { Study Area } \\
\text { (500m / } \\
500 m \text { / } \\
>500 m)\end{array}$ & $\begin{array}{c}\text { Radius of } \\
\text { the } \\
\text { Applied } \\
\text { Study } \\
\text { Area (m) } \\
\end{array}$ \\
\hline 1 & $\begin{array}{l}6^{\circ} 53^{\prime} 59.8^{\prime \prime} \mathrm{N} \\
79^{\circ} 51^{\prime} 21.6^{\prime \prime} \mathrm{E}\end{array}$ & $\begin{array}{l}\text { No: } 45 \text {, Alfred House Gardens, } \\
\text { Colombo } 3\end{array}$ & $\begin{array}{l}\text { Luxury } \\
\text { apartment } \\
\text { complex }\end{array}$ & $15,110.00$ & $<500$ & 270 \\
\hline 2 & $\begin{array}{c}6^{\circ} 49^{\prime} 51.4^{\prime \prime} \mathrm{N} \\
80^{\circ} 59^{\prime} 18.9^{\prime \prime} \mathrm{E}\end{array}$ & No: 7, Welimada Road, Bandarawela & Supermarket & $5,785.16$ & $<500$ & 240 \\
\hline
\end{tabular}




\begin{tabular}{|c|c|c|c|c|c|c|}
\hline 3 & $\begin{array}{c}6^{\circ} 58^{\prime} 13.5^{\prime \prime N} \\
80^{\circ} \mathrm{OO}^{\prime} 36.3^{\prime \prime} \mathrm{E}\end{array}$ & No: 4/B, Kanduboda, Delgoda & Hotel & $15,060.00$ & 500 & 500 \\
\hline 4 & $\begin{array}{l}6^{\circ} 42^{\prime} 42.3^{\prime \prime} \mathrm{N} \\
79^{\circ} 59^{\prime} 09.9^{\prime \prime} \mathrm{E}\end{array}$ & $\begin{array}{l}\text { No: 50, Panadura Road, } \\
\text { Bandaragama }\end{array}$ & Supermarket & $1,160.00$ & $<500$ & 130 \\
\hline 5 & $\begin{array}{c}6^{\circ} 54^{\prime} 28.1^{\prime \prime} \mathrm{N} \\
80^{\circ} \mathrm{O} 4^{\prime} 34.9^{\prime \prime} \mathrm{E}\end{array}$ & $\begin{array}{l}\text { No: } 143 / 6 \text {, Hanwella Road, Pahala } \\
\text { Hanwella, Hanwella }\end{array}$ & Supermarket & $1,160.00$ & $<500$ & 190 \\
\hline 6 & $\begin{array}{l}7^{\circ} 12^{\prime} 15 \cdot 0^{\prime \prime N} \\
79^{\circ} 51^{\prime} 43.9^{\prime \prime E}\end{array}$ & $\begin{array}{l}\text { No: 339, Kimbulapitiya Road, Akkara } \\
\text { Panaha, Kimbulapitiya }\end{array}$ & Supermarket & $1,151.00$ & $>500$ & 740 \\
\hline 7 & $\begin{array}{l}7^{\circ} 10^{\prime} 39.5^{\prime \prime} \mathrm{N} \\
79^{\circ} 51^{\prime} 43.6^{\prime \prime E}\end{array}$ & $\begin{array}{l}\text { No: } 58 \text {, Negombo Road, Kurana, } \\
\text { Katunayake }\end{array}$ & Supermarket & $1,151.00$ & $<500$ & 470 \\
\hline 8 & $\begin{array}{c}6^{\circ} 31^{\prime} 22.9^{\prime \prime} \mathrm{N} \\
80^{\circ} 06^{\prime} 33.7^{\prime \prime} \mathrm{E} \\
\end{array}$ & No: 185 , Kalutara Road, Matugama & Supermarket & $1,137.20$ & $>500$ & 540 \\
\hline 9 & $\begin{array}{l}6^{\circ} 45^{\prime} 48.5^{\prime \prime} \mathrm{N} \\
79^{\circ} 53^{\prime} 50.1^{\prime \prime} \mathrm{E}\end{array}$ & No: 350/13, Gorakana, Panadura & Hotel & $6,169.40$ & $<500$ & 120 \\
\hline 10 & $\begin{array}{l}6^{\circ} 49^{\prime} 53 \cdot 4^{\prime \prime} \mathrm{N} \\
80^{\circ} 59^{\prime} 16.2^{\prime \prime} \mathrm{E}\end{array}$ & $\begin{array}{l}\text { Jummah Masjid, Welimada Road, } \\
\text { Bandarawela }\end{array}$ & Mosque & $2,892.70$ & $>500$ & 560 \\
\hline 11 & $\begin{array}{l}6^{\circ} 50^{\prime} 20.3^{\prime \prime N} \\
79^{\circ} 58^{\prime} 43.9^{\prime \prime} \mathrm{E}\end{array}$ & $\begin{array}{l}\text { No: 1285, High Level Road, } \\
\text { Makubura, Kottawa }\end{array}$ & Supermarket & $1,031.22$ & $<500$ & 140 \\
\hline 12 & $\begin{array}{l}6^{\circ} 49^{\prime} 54.0^{\prime \prime N} \\
80^{\circ} 59^{\prime} 11.7^{\prime \prime} \mathrm{E}\end{array}$ & No: 21, Welimada Road, Bandarawela & Supermarket & $1,140.30$ & $>500$ & 750 \\
\hline 13 & $\begin{array}{l}6^{\circ} 53^{\prime} 45 \cdot 0^{\prime \prime N} \\
79^{\circ} 56^{\prime} 31.7^{\prime \prime} \mathrm{E}\end{array}$ & $\begin{array}{l}\text { No: 427, Siriwedapura, Akuregoda, } \\
\text { Battaramulla }\end{array}$ & Supermarket & 1185.56 & $<500$ & 360 \\
\hline 14 & $\begin{array}{l}6^{\circ} 57^{\prime} \mathrm{O} 7 \cdot 9^{\prime \prime} \mathrm{N} \\
80^{\circ} 12^{\prime} 52.7^{\prime \prime} \mathrm{E}\end{array}$ & $\begin{array}{l}\text { No: } 78 \text {, Yatiyanthota Road, } \\
\text { Avissawella }\end{array}$ & $\begin{array}{l}\text { Commercial \& } \\
\text { Office Building }\end{array}$ & $11,516.89$ & 500 & 500 \\
\hline 15 & $\begin{array}{l}7^{\circ} \mathrm{O} 4^{\prime} 32.1^{\prime \prime N} \\
80^{\circ} 00^{\prime} 50.7^{\prime \prime} \mathrm{E}\end{array}$ & No: 03, Miriswatta, Gampaha & Supermarket & $1,069.47$ & $<500$ & 340 \\
\hline 16 & $\begin{array}{l}7^{\circ} \mathrm{O} 7^{\prime} 37.0^{\prime \prime} \mathrm{N} \\
80^{\circ} 04^{\prime} 16.1^{\prime \prime E} \\
\end{array}$ & No: 120, Thihariya, Kalagedihena & Supermarket & 1098.8 & $>500$ & 620 \\
\hline 17 & $\begin{array}{l}6^{\circ} 54^{\prime} 16.0 " \mathrm{~N} \\
79^{\circ} 54^{\prime} 48.8^{\prime \prime} \mathrm{E}\end{array}$ & $\begin{array}{l}\text { Sethsiripaya Premises (Sethsiripaya } \\
\text { Stage III), Battaramulla }\end{array}$ & $\begin{array}{l}\text { High-Rise Office } \\
\text { Complex - } \\
\text { "Sethsiripaya } \\
\text { Stage-III" }\end{array}$ & $123,909.20$ & $<500$ & 350 \\
\hline 18 & $\begin{array}{l}6^{\circ} 54^{\prime} 35 \cdot 0^{\prime \prime} \mathrm{N} \\
79^{\circ} 51^{\prime} 01.4^{\prime \prime} \mathrm{E} \\
\end{array}$ & $\begin{array}{l}\text { No: } 271, \& 271 / 1, \text { Galle Road, } \\
\text { Colombo } 3\end{array}$ & Supermarket & 567.64 & $<500$ & 300 \\
\hline 19 & $\begin{array}{l}6^{\circ} 52^{\prime} 19.0^{\prime \prime} \mathrm{N} \\
79^{\circ} 51^{\prime} 45.9^{\prime \prime} \mathrm{E} \\
\end{array}$ & $\begin{array}{l}\text { No: } 12,12 \mathrm{~A}, 14 \text {, Rudra Mawatha, } \\
\text { Colombo o6 }\end{array}$ & Apartment & 14,160 & $>500$ & 570 \\
\hline 20 & $\begin{array}{c}6^{\circ} 51^{\prime} 41.9^{\prime \prime} \mathrm{N} \\
79^{\circ} 53^{\prime} 41.2^{\prime \prime} \mathrm{E}\end{array}$ & $\begin{array}{l}\text { No: 68, Pepiliyana Road, } \\
\text { Gangodawila, Nugegoda }\end{array}$ & Supermarket & 754 & $<500$ & 410 \\
\hline 21 & $\begin{array}{l}6^{\circ} 53^{\prime} 34.2^{\prime \prime N} \\
79^{\circ} 53^{\prime} 18.4^{\prime \prime} \mathrm{E}\end{array}$ & $\begin{array}{l}\text { No: o3, Swarna Place, Nawala Road, } \\
\text { Rajagiriya }\end{array}$ & Office Building & 4,197 & 500 & 500 \\
\hline 22 & $\begin{array}{l}6^{\circ} \mathrm{O} 2^{\prime} 52.9^{\prime \prime} \mathrm{N} \\
80^{\circ} 13^{\prime} 18.2^{\prime \prime} \mathrm{E}\end{array}$ & $\begin{array}{l}\text { No: } 314 \mathrm{C}, 316,318 \text {, Sirimbura road, } \\
\text { Dangedara, Galle }\end{array}$ & Supermarket & $1,082.40$ & $<500$ & 50 \\
\hline 23 & $\begin{array}{l}7^{\circ} \mathrm{O} 4^{\prime} 57.5^{\prime \prime N} \\
79^{\circ} 53^{\prime} 19.6^{\prime \prime} \mathrm{E} \\
\end{array}$ & $\begin{array}{l}\text { No: } 32 \text {, Gamameda Road, Thudella, } \\
\text { Ja-Ela }\end{array}$ & Storage Building & 55.83 & $<500$ & 250 \\
\hline 24 & $\begin{array}{c}6^{\circ} 47^{\prime} 34.4^{\prime \prime N} \\
79^{\circ} 56^{\prime} 48.8^{\prime \prime} \mathrm{E} \\
\end{array}$ & No: 1059, Horana Road, Kesbewa & Supermarket & 1,186 & $>500$ & 710 \\
\hline 25 & $\begin{array}{c}5^{\circ} 56^{\prime} 44.6^{\prime \prime} \mathrm{N} \\
80^{\circ} 30^{\prime} 54.5^{\prime \prime} \mathrm{E}\end{array}$ & $\begin{array}{l}\text { No: 259, Sri Sunanda Mawatha, } \\
\text { Walgama, Matara }\end{array}$ & Private School & $2,677 \cdot 74$ & $<500$ & 480 \\
\hline 26 & $\begin{array}{c}7^{\circ} 10^{\prime} 15 \cdot 3^{\prime \prime} \mathrm{N} \\
79^{\circ} 56^{\prime} 35 \cdot 9^{\prime \prime} \mathrm{E}\end{array}$ & $\begin{array}{l}\text { No: 125/1, Katunayake Road, } \\
\text { Minuwangoda }\end{array}$ & Supermarket & $4,094.70$ & $<500$ & 310 \\
\hline 27 & $\begin{array}{l}7^{\circ} 13^{\prime} 42.8^{\prime \prime N} \\
79^{\circ} 54^{\prime} 43 \cdot 7^{\prime \prime} \mathrm{E} \\
\end{array}$ & $\begin{array}{l}\text { East Field Estate, Kaluwarippuwa } \\
\text { East Village, Negombo Road, } \\
\text { Miriswatta }\end{array}$ & $\begin{array}{l}\text { Cargills } \\
\text { Distribution } \\
\text { Centre }\end{array}$ & 26,300 & $<500$ & 300 \\
\hline 28 & $\begin{array}{l}6^{\circ} 46^{\prime} 49.6^{\prime \prime N} \\
79^{\circ} 52^{\prime} 58.3^{\prime \prime} \mathrm{E} \\
\end{array}$ & No: 776, Galle Road, Moratuwa & Supermarket & $1,703.83$ & $<500$ & 480 \\
\hline 29 & $\begin{array}{l}6^{\circ} 50^{\prime} 10.3^{\prime \prime} \mathrm{N} \\
79^{\circ} 52^{\prime} \mathrm{O} \cdot 5^{\prime \prime} \mathrm{E} \\
\end{array}$ & No: 282, Galle Road, Mt. Lavinia & Supermarket & $3,228.60$ & $<500$ & 380 \\
\hline 30 & $\begin{array}{l}6^{\circ} 54^{\prime} 47.7^{\prime \prime N} \\
79^{\circ} 56^{\prime} \mathrm{O} .7^{\prime \prime} \mathrm{E} \\
\end{array}$ & No. 30, Pipe Road, Battaramulla & Apartment & $27,722.90$ & $<500$ & 400 \\
\hline 31 & $\begin{array}{c}7^{\circ} 13^{\prime} 48.6^{\prime \prime N} \\
79^{\circ} 51^{\prime} \mathrm{O} .3^{\prime \prime E} \\
\end{array}$ & $\begin{array}{l}\text { No: } 254 / 1 \text {, Chilaw Road, Kattuwa, } \\
\text { Negambo }\end{array}$ & Supermarket & 1190.7 & $<500$ & 100 \\
\hline 32 & $\begin{array}{c}6^{\circ} 56^{\prime} 31.1^{\prime \prime N} \\
79^{\circ} 51^{\prime} 56.6 " \mathrm{E} \\
\end{array}$ & $\begin{array}{l}\text { No: } 38 \text {, M. Vincent Perera Mawatha, } \\
\text { Colombo } 14\end{array}$ & Retail Complex & 3,158 & $<500$ & 260 \\
\hline 33 & $\begin{array}{l}7^{\circ} 35^{\prime} \mathrm{O} 1.4^{\prime \prime N} \\
79^{\circ} 47^{\prime} 49.1^{\prime \prime E}\end{array}$ & Lansiya Watta, Chilaw & Supermarket & $1,082.40$ & $<500$ & 20 \\
\hline 34 & $\begin{array}{c}6^{\circ} 58^{\prime} 41.1^{\prime \prime N} \\
79^{\circ} 53^{\prime} 19.5^{\prime \prime E} \\
\end{array}$ & No: 85, Negombo Road, Wattala & Supermarket & $1114 \cdot 3$ & $<500$ & 130 \\
\hline 35 & $\begin{array}{l}6^{\circ} 48^{\prime} 56.3^{\prime \prime N} \\
80^{\circ} 57^{\prime} 47.5^{\prime \prime} \mathrm{E} \\
\end{array}$ & $\begin{array}{l}\text { Boer Road, Range View, Kahagolla, } \\
\text { Diyathalawa }\end{array}$ & $\begin{array}{l}\text { Fuel Filling } \\
\text { Station }\end{array}$ & 672.62 & $>500$ & 520 \\
\hline 36 & $\begin{array}{l}6^{\circ} 55^{\prime} \mathrm{O} 2.5^{\prime \prime} \mathrm{N} \\
79^{\circ} 51^{\prime} 39.2^{\prime \prime} \mathrm{E} \\
\end{array}$ & $\begin{array}{l}\text { No: 69, Hyde Park Corner, Colombo } \\
2\end{array}$ & Supermarket & $13,154.00$ & $<500$ & 320 \\
\hline 37 & $\begin{array}{l}6^{\circ} 50^{\prime} 55 \cdot 3^{\prime \prime N} \\
79^{\circ} 52^{\prime} 39.1^{\prime \prime} \mathrm{E} \\
\end{array}$ & $\begin{array}{l}\text { No.15, Rohini Road, Nikape, } \\
\text { Dehiwala }\end{array}$ & Apartment & $9,766.50$ & $<500$ & 430 \\
\hline 38 & $\begin{array}{l}6^{\circ} 59^{\prime} \mathrm{O} 9.3^{\prime \prime N} \\
79^{\circ} 53^{\prime} 18.3^{\prime \prime} \mathrm{E} \\
\end{array}$ & No.331, Negombo Road, Wattala & Supermarket & $2,772.42$ & $>500$ & 660 \\
\hline 39 & $\begin{array}{l}6^{\circ} 50^{\prime} 37.9^{\prime \prime} \mathrm{N} \\
79^{\circ} 55^{\prime} \mathrm{O} 7.8^{\prime \prime} \mathrm{E} \\
\end{array}$ & No. 24, Lake Road, Maharagama & $\begin{array}{l}\text { Hirdaramani } \\
\text { Discovery Lab }\end{array}$ & $2,956.08$ & $<500$ & 450 \\
\hline
\end{tabular}




\begin{tabular}{|c|c|c|c|c|c|c|}
\hline 40 & $\begin{array}{l}6^{\circ} 48^{\prime} 09.0^{\prime \prime} \mathrm{N} \\
79^{\circ} 53^{\prime} 17.2^{\prime \prime} \mathrm{E}\end{array}$ & No: 339 \& 3, Galle Road, Moratuwa & $\begin{array}{l}\text { Mixed } \\
\text { Development }\end{array}$ & $7,090.50$ & $>500$ & 540 \\
\hline 41 & $\begin{array}{l}6^{\circ} 57^{\prime} 10.2^{\prime \prime N} \\
79^{\circ} 55^{\prime} 47.4^{\prime \prime} \mathrm{E}\end{array}$ & $\begin{array}{l}\text { No. 356, Biyagama Road, Gonawela, } \\
\text { Kelaniya }\end{array}$ & Supermarket & $1,098.80$ & $<500$ & 230 \\
\hline 42 & $\begin{array}{c}5^{\circ} 57^{\prime} 15 \cdot 3^{\prime \prime N} \\
80^{\circ} 32^{\prime} \mathrm{O} 1.8^{\prime \prime} \mathrm{E}\end{array}$ & $\begin{array}{l}\text { No. 53B, Sri Rathanapala Mawatha, } \\
\text { Matara }\end{array}$ & $\begin{array}{l}\text { Mixed } \\
\text { Development }\end{array}$ & 821.69 & $<500$ & 470 \\
\hline 43 & $\begin{array}{l}6^{\circ} 52^{\prime} \mathrm{O} 4.8^{\prime \prime} \mathrm{N} \\
79^{\circ} 55^{\prime} 48.3^{\prime \prime} \mathrm{E}\end{array}$ & $\begin{array}{l}\text { 31A, Hospital Road, Sri } \\
\text { Jayawardenapura }\end{array}$ & $\begin{array}{l}\text { Banquet Hall } \\
\text { Complex }\end{array}$ & $13,608.70$ & $<500$ & 320 \\
\hline 44 & $\begin{array}{c}6^{\circ} 56^{\prime} 10.5^{\prime \prime N} \\
81^{\circ} 09^{\prime} 42.7^{\prime \prime} \mathrm{E}\end{array}$ & Batticaloa Road, Passara & Fuel Station & $827 \cdot 3$ & $<500$ & 330 \\
\hline 45 & $\begin{array}{l}6^{\circ} \mathrm{O} 1^{\prime} 11.2^{\prime \prime} \mathrm{N} \\
80^{\circ} 14^{\prime} 52.1^{\prime \prime} \mathrm{E}\end{array}$ & No: 134, Matara Road, Unawatuna & Supermarket & 625.42 & $<500$ & 50 \\
\hline 46 & $\begin{array}{l}7^{\circ} 13^{\prime} 10.3^{\prime \prime N} \\
79^{\circ} 52^{\prime} \mathrm{O} 6.5^{\prime \prime} \mathrm{E}\end{array}$ & $\begin{array}{l}\text { Dawatagahawatte, } \\
\text { Thimbirigaskatuwa, Negombo }\end{array}$ & Private School & $2,877.18$ & $>500$ & 590 \\
\hline 47 & $\begin{array}{l}6^{\circ} 51^{\prime} 18.9^{\prime \prime} \mathrm{N} \\
79^{\circ} 53^{\prime} 29.4^{\prime \prime} \mathrm{E}\end{array}$ & No: 338 , Colombo Road, Pepiliyana & Supermarket & $1,270.90$ & $<500$ & 220 \\
\hline 48 & $\begin{array}{c}6^{\circ} 51^{\prime} 19.3^{\prime \prime N} \\
79^{\circ} 54^{\prime} 44.7^{\prime \prime} \mathrm{E}\end{array}$ & $\begin{array}{l}\text { No: 443, High Level Road, Nawinna, } \\
\text { Maharagama }\end{array}$ & $\begin{array}{l}\text { Assembly of God } \\
\text { Church }\end{array}$ & $1,432.65$ & $>500$ & 520 \\
\hline 49 & $\begin{array}{l}6^{\circ} 52^{\prime} 10.4^{\prime \prime} \mathrm{N} \\
79^{\circ} 55^{\prime} 47.2^{\prime \prime} \mathrm{E} \\
\end{array}$ & $\begin{array}{l}\text { No: 31, Hospital Road, Madiwela, } \\
\text { Thalapathpitiya }\end{array}$ & Apartment & $48,099.23$ & $<500$ & 460 \\
\hline 50 & $\begin{array}{l}6^{\circ} \mathrm{O} 4^{\prime} \mathrm{O} 1.8^{\prime \prime} \mathrm{N} \\
80^{\circ} 13^{\prime} 24.3^{\prime \prime} \mathrm{E} \\
\end{array}$ & $\begin{array}{l}\text { No: 572, Hirimbura Road, Karapitiya, } \\
\text { Galle }\end{array}$ & Supermarket & 660.26 & $<500$ & 320 \\
\hline
\end{tabular}

Figure 7 clearly shows the different radius values for the study area between the TIA guidelines and at practice. The scatter graph shows the different radius values of the study areas at the practice and dotted straight line shows 500m radius under the TIA guideline. Accordingly, very few studies have undertaken the 500m or a closer radius of the study area for their analysis. Besides, according to the 50 TIA case studies, most of them had been carried out with either more than or less than the 500m radius of the study area for traffic impact analysis. Hence, there is a strong gap between the true catchment areas and applied catchment areas in these TIA studies.

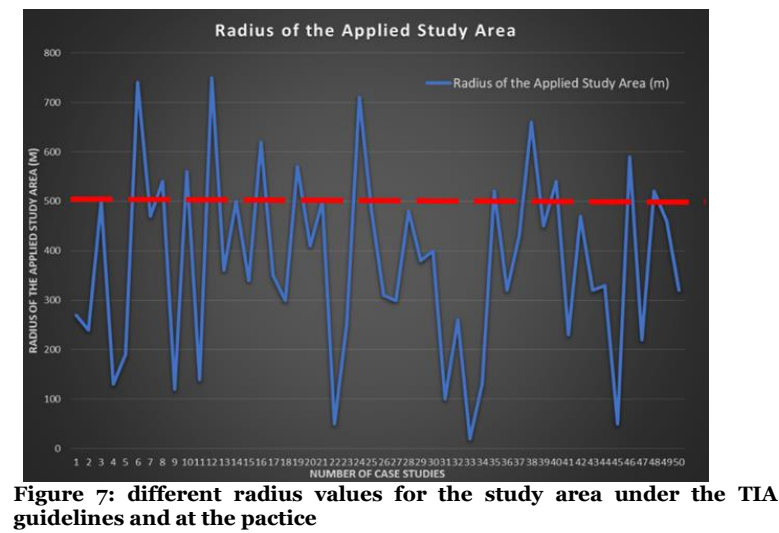

According to the results of the descriptive statistic analysis for the floor area of each development from BM SPSS Statistics software, the mean value is $7874.7638 \mathrm{sqm}$. There is a high range between maximum and minimum radiuses which is $123853.37 \mathrm{sqm}$. This data set which has a high variance is $357466269.668 \mathrm{sqm}$. A high standard deviation is in the data set, which is $18906.77841 \mathrm{sqm}$. Further, this data set is widely spread out.

According to the results of the descriptive statistics analysis for the radius of the applied study area from BM SPSS Statistics software, the middle value of the data set (median) is 370m. There are multiple modes in this data list and the smallest mode value is $320 \mathrm{~m}$ and maximum mode value $500 \mathrm{~m}$. The applied average radius of the study area (mean) for each development is $378 \mathrm{~m}$. Thus, the maximum radius value of the applied study area is $750 \mathrm{~m}$ and the minimum radius value of the applied study area is $20 \mathrm{~m}$. Thus, there is a high range between maximum and minimum radiuses which is $730 \mathrm{~m}$. This data set has a high variance, which is $33995.918 \mathrm{~m}$. A high standard deviation is in the data set, which is $184.37982 \mathrm{~m}$. Furthermore, this data set is widely spread out. Therefore, the application of a fixed radius for different development types misdirects the planning decisions in a city. 
Table 2: Results of the Descriptive Statistics Analysis

Statistics

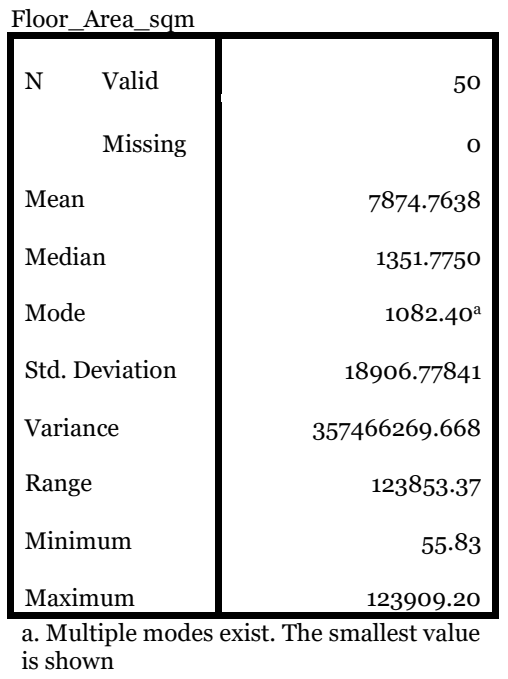

Statistics

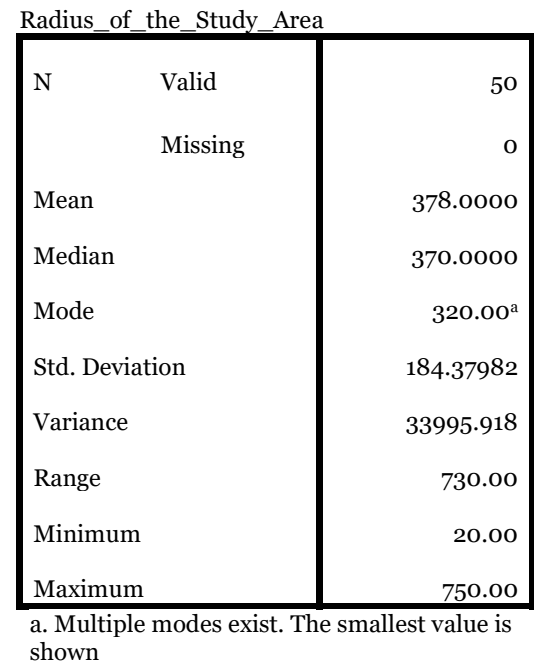

\section{Conclusion}

There are different problematic areas in TIA guidelines and it may negatively affect the future of town planning. Cooley, Gruyter, \& Delbosc (2016), argue that although many national TIA guidelines are available, there is no proper scope to practice well. Accordingly, although planning authorities of different countries recommend adopting the TIA guidelines to prepare TIAs to assess the transport impacts (Abley, Durdin, \& Douglass, 2010), many technical and operational disparities have been identified in the TIA guidelines (van Rensburg \& van As, 2004). One of them is the absence of a flexible study area (Ponnurangama \& Umadevib, 2014). It means that there is a gap between the regulatory boundary and the adopted boundaries at the practice in TIAs. Different kinds of literature and analytical results prove undoubtedly that there is a requirement of a flexible study area for traffic impact assessments of each distinctly unique development. Hence, this study not only shows the way to adopt or develop a new technique to define the true catchment area for traffic impact assessments, but has also helped to rectify reasonable decision making in the transport planning sector.

\section{References}

Abley, S., Durdin, P., \& Douglass, M. (2010). Integrated transport assessment guidelines. Retrieved July 14, 2019, from https://www.nzta.govt.nz/assets/resources/research/reports/422/docs/422.pdf ACT Government \& Transport Canberra and City Services (TCCS). (August 2016). Guidelines for Transport Impact Assessment. Australia. Retrieved July 16, 2019, from https://www.tccs.act.gov.au/tams_files/search?query=traffic+impacts Araldi, A. (2020). Towards an Integrated Methodology for Model and Variable Selection Using Count Data: An Application to Micro-Retail Distribution in Urban Studies. doi:10.3390/urbansci4020021 Arballo, C. (December 2016). Transportation Impact Study Guidelines. City of Los Angeles: City of Los Angeles Department of Transportation (LADOT). Retrieved July 1, 2019, from https://ladot.lacity.org/sites/g/files/wph266/f/COLA-TISGuidelines-010517.pdf

Azra, N., \& Hoque, M. S. (2014, September 30). Implementation of Traffic Impact Assessment in Developing Countries: Case Study of Bangladesh. International Journal of Transport, Highways \& Railways Engineering, 1(3), 7-11. Retrieved August 18, 2019, from http://journals.theired.org/assets/pdf/20170419_113601.pdf Bihar State Road Development Corporation Limited, Government of Bihar for the Asian Development Bank. (2016). Environmental Impact Assessment (Draft). Bihar, India. Retrieved August 8, 2019, from https://www.adb.org/sites/default/files/linked-documents/48373-007-eiaab.pdf Birkin, M., Clarke, G., \& Clarke, M. (2010, October 18). Refining and Operationalizing Entropy-Maximizing Models for Business Applications. A 40th Anniversary Celebration of Alan Wilson, 1970, Entropy in Urban and Regional Modelling, 42(4), 422-445. doi:

Bureau of Traffic Operations. (2019). Traffic Impact Analysis Guidelines. Wisconsin, USA. Retrieved June 26, 2019, from https://wisconsindot.gov/dtsdManuals/traffic-ops/manuals-and-standards/tiaguide.pdf Cabral, P., Augusto, G., Tewolde, M., \& Araya, Y. (2013, November 27). Entropy in Urban Systems. 15(12). doi:10.3390/e15125223 
California Department of Transportation (Caltrans). (December 2002). Guide for the Preparation of Traffic Impact Studies. State Of California, United State. Retrieved July 23, 2019, from https://nacto.org/wpcontent/uploads/2015/04/guide_preparation_traffic_impact_studies_caltrans.pdf City of Aspen. (n.d.). Transportation Impact Analysis Guidelines. City of Aspen, Colorado, USA. Retrieved July 17, 2019, from https://www.cityofaspen.com/DocumentCenter/View/2208/TIA-Guidelines City of Edmonton. (2016). Transportation Impact Assessment Guidelines. Edmonton, Alberta, Canada. Retrieved July 22, 2019, from https://www.edmonton.ca/city_government/documents/RoadsTraffic/TIA_Guidelines_2016.pdf Community Development Department. (2019). Procedures For Traffic Impact Analysis (TIA). City of Visalia, California, USA. Retrieved July 15, 2019, from https://www.visalia.city/civicax/filebank/blobdload.aspx?BlobID=41216 Cooley, K., Gruyter, C. D., \& Delbosc, A. (2016). A best practice evaluation of traffic impact assessment guidelines in Australia and New Zealand. Australasian Transport Research Forum 2016. Retrieved June 30, 2019, from https://www.atrf.info/papers/2016/files/ATRF2016_Full_papers_resubmission_155.pdf Coombes, M., \& Casado Diaz, J. (2005). The evolution of Local Labour Market Areas in contrasting regions. 45th Congress of the European Regional Science Association. Retrieved May 02, 2020, from

https://www.researchgate.net/publication/277138549_The_evolution_of_Local_Labour_Market_Areas_in_contrastin g_regions

Delloye, J. (2018, April). Urban Morphodynamics : Reconciling Location Theory and Complex Systems. Retrieved from https://www.researchgate.net/profile/Justin_Delloye/publication/326667016_Urban_Morphodynamics_Reconciling_ Location_Theory_and_Complex_Systems/links/5b8806b04585151fd13c8a9f/Urban-Morphodynamics-ReconcilingLocation-Theory-and-Complex-Systems.pdf?origin $=p$

Department for Regional Development (DRD) \& Department of the Environment (DOE). (November 2006). Guidelines for Development Proposals in Northern Ireland. Planning Policy Statement 13: Transportation and Land Use and Planning Policy Statement 3: Access, Movement and Parking, Northern Ireland, United Kingdom. Retrieved JUly 15, 2019, from https://www.planningni.gov.uk/transport-assessment.pdf

Department of Planning. (August 2016). Transport Impact Assessment Guidelines. Perth, Australia: Western Australian Planning Commission. Retrieved July 18, 2019, from https://www.dplh.wa.gov.au/getmedia/913e74ea-0290-4982aco9-83d27951e4c2/GD_Transport_impact_assessment_vol5pdf

Department of Transport and main roads. (September 2017). Guide to Traffic Impact Assessment. Queensland, Australia. Retrieved July 18, 2019, from https://www.tmr.qld.gov.au/-/media/busind/techstdpubs/Road-planning-anddesign/Guidelines-to-Traffic-Impact-Assessment/Guide-to-Traffic-Impact-Assessment.pdf?la=en

Department of Transport. (March 2007). Guidance on Transport Assessment. London, United Kingdom. Retrieved July 16, 2019, from

https://assets.publishing.service.gov.uk/government/uploads/system/uploads/attachment_data/file/263054/guidance -transport-assessment.pdf

Department of Transport. (November 2009). Transportation Impact Study Guidelines. Emirate of Abu Dhabi , United Arab Emirates. Retrieved July 23, 2019, from http://nacv.pbworks.com/f/DoT+TIS+Guidelines_1.1.pdf Dolega, L., Pavlis, M., \& Singleton, A. (2015, September 05). Estimating attractiveness, hierarchy and catchment area extents for a national set of retail centre agglomerations. Journal of Retailing and Consumer Services, 78-90. doi:http://dx.doi.org/10.1016/j.jretconser.2015.08.013

Division of the Town of Caledon. (2017). Transportation Impact Studies - Terms of Reference and Guideline. Town of Caledon, Ontario, Canada . Retrieved July 23, 2019, from

https://www.caledon.ca/en/townhall/resources/Transportation-Impact-Studies-TOR-Guidelines.pdf

Dolega, L., Pavlis, M., \& Singleton, A. (2015, September 05). Estimating attractiveness, hierarchy and catchment area extents for a national set of retail centre agglomerations. Journal of Retailing and Consumer Services, 78-90.

doi:http://dx.doi.org/10.1016/j.jretconser.2015.08.013

Dramowicz, E. (2005, July 03). Retail Trade Area Analysis Using the Huff Model. Retrieved from

https://www.directionsmag.com/article/3207

Engineering and Capital Infrastructure Services Infrastructure, Development \& Enterprise. (April 2016). Traffic Impact Study Guidelines. Guelph, Ontario, Canada: Engineering and Capital Infrastructure Services Infrastructure, Development \& Enterprise. Retrieved July 22, 2019, from https://guelph.ca/wpcontent/uploads/TrafficImpactStudyGuidelines.pdf

Fang, Y., Mao, J., Liu, Q., \& Huang, J. (2020). Exploratory space data analysis of spatial patterns of large-scale retail commercial facilities: The case of Gulou District, Nanjing, China. ScienceDirect. doi:10.1016/j.foar.2020.02.002 Government of India Ministry of Environment \& Forests, New Delhi, India. (n.d.). The Environmental Impact Assessment Notification, 1994 (As Amended on 4-5-94) . New Delhi, India. Retrieved August 18, 2019, from http://extwprlegs1.fao.org/docs/pdf/ind4656.pdf

Guldmann, J.-M. (1999). Competing destinations and intervening opportunities interaction models of inter-city telecommunication flows. Retrieved May 01, 2020, from

https://www.researchgate.net/publication/24060908_Competing_destinations_and_intervening_opportunities_inter action_models_of_inter-city_telecommunication_flows

Huff, D. (2003, October-December). Parameter Estimation in the Huff Model. pp. 34-36. Retrieved April 27, 2020, from https://www.esri.com/news/arcuser/1003/files/huff.pdf

IBI Group and City of London. (2012 ). Transportation Impact Assessment Guidelines. City of London, United Kingdom. Retrieved July 17, 2019, from https://www.london.ca/residents/Roads-Transportation/Transportation-

Planning/Documents/Transportation_Impact_Assessment_Guidelines.pdf

Indiana Department of Transportation. (May 2015 ). Applicant's Guide to Traffic Impact Studies . Indiana, Unite State.

Retrieved June 22, 2019, from

https://www.in.gov/indot/files/Permits_ApplicantsGuidetoTrafficImpactStudy_2015.pdf 
Khade, O. S., Khode, B. V., \& Bhakhtyapuri, V. K. (2017, March). Evaluation of Traffic Impact on Road Network due to New Commercial Development. IJSTE - International Journal of Science Technology \& Engineering, 3(9). Retrieved August 15, 2019, from http://www.ijste.org/articles/IJSTEV3I9221.pdf

Land Transport Authority. (2011). Guidelines for Preparation of Traffic Impact Assessment Reports. Singapore: Land Transport Authority. Retrieved June 20, 2019, from

https://www.corenet.gov.sg/einfo/Uploads/Circulars/CLTA111230.pdf

Land Transport Authority. (2017). Transport Impact Assessment Guidelines for Developments. Singapore: Land Transport Authority. Retrieved July 22, 2019, from

https://www.lta.gov.sg/content/dam/ltaweb/corp/Industry/files/COP-

Appendices/Transport\%20Impact\%20Assessment\%20Guidelines_28\%20Sep\%202017.pdf

Lenormand, M., Bassolas, A., \& Ramasco, J. (2016, January 18). Systematic comparison of trip distribution laws and models. doi:probaarXiv:1506.04889v3

Leszczyc, P. P., Sinha, A., \& Sahgal, A. (2004, December). The Effect of Multi-Purpose Shopping on Pricing and Location Strategy for Grocery Stores. 85-99. doi:10.1016/j.jretai.2004.04.006

May, L. W., Rahman, R. A., Hassin, M. F., Diah, J. M., Mashros, N., Abdullah, M. E., \& Masirin , M. I. ( 2019, May). An Overview of the Practice of Traffic Impact Assessment in Malaysia. International Journal of Engineering and Advanced Technology (IJEAT), 8(5C), 916-921. doi:10.35940/ijeat.E1130.0585C19

Ministry of Public Infrastructure \& Land Transport. (2015). Traffic Impact Assessment Guidelines. Retrieved August 18, 2019, from http://publicinfrastructure.govmu.org/English//DOCUMENTS/REVISED\%20TIA\%20GUIDELINES.PDF Ministry of Public Infrastructure \& Land Transport. (November 2015). Traffic Impact Assessment Guideline. Land Transport Division of Ministry of Public Infrastructure \& Land Transport. Port Louis, Mauritius: Ministry of Public Infrastructure \& Land Transport. Retrieved July 10, 2019, from http://publicinfrastructure.govmu.org/English//DOCUMENTS/REVISED\%2oTIA\%20GUIDELINES.PDF National Roads Authority. (2014). Traffic and Transport Assessment Guidelines. Dublin, Ireland. Retrieved June 26, 2019, from https://www.tii.ie/tii-library/land-use-planning/Transport-Assessment-GuidelinesMay2014.pdf National Roads Authority. (2014). Traffic and Transport Assessment Guidelines. Dublin, Ireland. Retrieved June 26, 2019, from https://www.tii.ie/tii-library/land-use-planning/Transport-Assessment-GuidelinesMay2014.pdf New Prague City Council. (September, 2010). Traffic Impact Study Guidelines \& Process. City of New Prague, Minnesota, USA. Retrieved July 10, 2019, from https://www.ci.new-prague.mn.us/vertical/sites/\%7BAD7ECB62-2C5E-4BAo-8F191426026AFA3E\%7D/uploads/\%7B09164999-DFCD-4643-9D13-97D02C8BA21C\%7D.PDF

Norwalk Transportation Management Plan. (n.d.). Transportation Impact \& Access Study Guidelines. City of Norwalk, California, USA. Retrieved July 22, 2019, from http://projects.vhb.com/norwalktmp/pdf/final/Chapter\%202-4\%20\%20Traffic\%20Impact\%20Guidelines.pdf

Office of Transportation (OOT). (2005). Guidelines for Development of Traffic Impact Reports. City of Berkeley, California. Retrieved July 2, 2019, from https://www.cityofberkeley.info/uploadedFiles/Public_Works/Level_3_-

General/TIRGuidelines_v.1_09-16-2005.pdf

PMK Associates, Inc. (July 2006). Traffic Impact Study Guidelines. City of Corona, Riverside County, California, United States: Public Works Department. Retrieved June 25, 2019, from

https://www.coronaca.gov/home/showdocument?id=1650

Ponnurangama, P., \& Umadevib, G. (2014). Traffic Impact Analysis (TIA) for Chennai IT Corridor. Retrieved July 23, 2019, from https://www.sciencedirect.com/science/article/pii/S2352146516306937

Pratt, M., Wright, J., Cockings, S., \& Sterland, I. (n.d.). Delineating retail conurbations: a rules-based algorithmic approach. Retrieved May 02, 2020, from https://eprints.soton.ac.uk/365504/1/Delineating\%2520Retail\%2520Conurbations_Pratt_et_al_JRCS_2014_21_667675.pdf

Purvis, B., Mao, Y., \& Robinson, D. (2019, January 12). Entropy and its Application to Urban Systems. Entropy 2019. doi:10.3390/e21010056

Sarkar, P. K., Maitri, V., \& Joshi, G. J. (2015). Transportation Planning - Principals, Practices and Policies.

Segal, D. B. (1998, November 19). Retail Trade Area Analysis: Concepts and New Approaches. Retrieved April 27, 2020, from https://www.directionsmag.com/article/4157

Stantec Consulting Ltd. (March 2005). Traffic Impact Assessment Guideline. Alberta, Canada : Alberta Infrastructure \& Transportation, Transportation \& Civil Engineering Division. Retrieved July 2, 2019, from http://www.transportation.alberta.ca/Content/docType329/Production/TIA_guideline.pdf

Sturley, C. (2016, September). A proof of concept agent-based model of consumer store choice behaviour. Retrieved April 30, 2020, from https://www.researchgate.net/publication/312919080_A_proof_of_concept_agentbased_model_of_consumer_store_choice_behaviour

Teodoro, R. V., \& Regidor, J. R. (2005). Traffic Impact Assessment for Sustainable Traffic Management and Transportation Planning in Urban Areas. Eastern Asia Society for Transportation Studies. Retrieved June 19, 2019, from https://s3.amazonaws.com/academia.edu.documents/42000749/2342.pdf?response-contentdisposition=inline\%3B\%2ofilename\%3DTraffic_Impact_Assessment_for_Sustainabl.pdf\&X-Amz-Algorithm=AWS4HMAC-SHA256\&X-Amz-Credential=AKIAIWOWYYGZ2Y53UL3A\%2F20190814\%2Fus-eastThe Corporation of the City of Mississauga. (n.d.). Traffic Impact Study Guidelines. City of Mississauga, Ontario, Canada. Retrieved July 22, 2019, from http://www.mississauga.ca/file/COM/Traffic-Impact-Study-Guidelines.pdf Town Council Of Buckeye. (December 2012). Traffic Impact Analysis. Town Of Buckeye, Arizona, USA. Retrieved July 13, 2019, from https://www.buckeyeaz.gov/home/showdocument?id=444

United Nations. (1999). Transport and Communications Bulletinfor Asia and the Pacific. Urban Transport in the Asian and Pacific Region, New York. Retrieved August 15, 2019, from

https://www.unescap.org/sites/default/files/bulletin68_fulltext.pdf\#page=7

Urban Development Authority. (2001). build Srilanka. Com. Retrieved March 11, 2019, from City of Colombo Development Plan: http://www.buildsrilanka.com/CDP/Index.htm 
Urban Development Authority. (2018). Terms of Reference for Traffic Impact Assessment. Traffic Impact Assessment Committee of Urban Development Authority, Sri Lanka.

Utah Department of Transportation. (2004). Traffic Impact Study Requirements. State of Utah, united State. Retrieved July 14, 2019, from https://nacto.org/docs/usdg/traffic_impact_study_guidelines\%20_utah_dot.pdf

Utah Department of Transportation. (February 2015). Traffic Impact Study Guidelines. State of Utah, united State.

Retrieved July 13, 2019, from https://www.udot.utah.gov/main/uconowner.gf?n=20390830840115233

Van Rensburg, J., \& van As, S. (2004, July 12 - 15 ). Issues with Traffic Impact Assessments. 23rd Southern African

Transport Conference (SATC 2004), 654-661. Retrieved March 14, 2020, from

https://pdfs.semanticscholar.org/7321/80abde4aaa221d7ad95fb303d6e8f820c52f.pdf

Weerasekera, K. S. (2011). Impact on Existing Transport Systems by Generated Traffic due to New Developments.

Retrieved June 25, 2019, from

https://www.google.com/search?q=Impact+on+Existing+Transport+Systems+by+Generated+Traffic+due+to+New+D

evelopments\&oq=Impact+on+Existing+Transport+Systems +by+Generated+Traffic+due+to+New+Developments\&aqs $=$ chrome..69i57.886joj7\&sourceid=chrome\&ie=UTF-8

Wegener, M., \& Fuerst, F. (2004, October). Land-Use Transport Interaction: State of the Art. SSRN Electronic Journal. doi:10.2139/ssrn.1434678

Weller, P. (2007, August). Transport impact guidelines for site development : Literature review. Land Transport New

Zealand Research Report 327 Part II. Retrieved July 14, 2019, from

https://www.nzta.govt.nz/assets/resources/research/reports/327/docs/327-part2.pdf

Wen, T.-H., Chin, B., \& Lai, P.-C. (2017, January ). Link Structure Analysis of Urban Street Networks for Delineating

Traffic Impact Areas. doi: 10.1007/978-3-319-46164-9_10

Wilson, A. (2010, October 18 ). Entropy in Urban and Regional Modelling: Retrospect and Prospect. doi:10.1111/j.

Works Emergency Services Department, Urban Development Services Department \& Toronto Transit Commission. (July 2003). Guidelines for the Preparation of Transportation Impact Studies. Toronto, Ontario, Canada. Retrieved July 16, 2019, from http://arris.ca/ arris2/ARCHIVE/traffic-impact-study-guidelines.pdf

York County Government. (November 2017). Traffic Impact Analysis Guidelines Methodology. York County, United State. Retrieved July 18, 2019, from https://www.yorkcountygov.com/DocumentCenter/View/644/Traffic-ImpactAnalysis-Guidelines-Methodology-PDF

Yu-Long P. \& Xiao-ning W. (2004). Determining method of traffic impact range of project. Retrieved July 10, 2019, from http://en.cnki.com.cn/Article_en/CJFDTotal-JYGC200403019.htm

Zheng, Y., Zhao, G., \& Liu, J. (2015, January). A Novel Grid Based K-Means Cluster Method for Traffic Zone Division. International Conference on Cloud Computing and Big Data in Asia. doi:10.1007/978-3-319-28430-9_13 